\title{
A CASE REPORT OF A PERICARDIAL EXTRAOSSEOUS EWING SARCOMA
}

Stavrou Vasileios ${ }^{1}$, Gotsi Irini ${ }^{1}$, Christakou Eleni ${ }^{1}$, Kourtesis Antonios ${ }^{2}$, Stefanaki Kalliopi ${ }^{3}$, Polychronopoulou Sofia ${ }^{4}$, Barbaresou Charikleia ${ }^{1}$

espnIC $\quad$ 'Pediatric Intensive Care Unit, Aghia Sophia Children's Hospital, Athens, Greece

${ }^{2}$ Department of Cardiothoracic Surgery, Aghia Sophia Children's Hospital, Athens, Greece

${ }^{3}$ Pathology Department, Aghia Sophia Children's Hospital, Athens, Greece

${ }^{4}$ Department of Paediatric Haematology-Oncology , Aghia Sophia Children's Hospital, Athens, Greece

picu@paidon-agiasophia.gr

\section{BACKGROUND}

Ewing's sarcoma is a poorly differentiated, highly malignant tumor. It arises primarily from bones and extraosseous origin is rare. Commonly affected extraosseous sites include the lower extremities, head and pelvis. Only few cases of primary pericardial or cardiac involvement have been described.

\section{METHODS}

We present a 8,5 year-old boy with pericardial extraosseous Ewing sarcoma (EES) admitted to PICU due to hemopericardium and polyserositis.

\section{RESULTS}

The patient was originally admitted to a local hospital due to weight loss, fever, respiratory distress, vomiting and abdominal pain. Initial US showed ascites, pericardial and bilateral pleural effusions. Chest

CT scan revealed a pericardial mass and pericardial effusion causing tamponade. An emergency pericardiocentesis was carried out, which drained $700 \mathrm{ml}$ of hemorrhagic fluid. Subsequently, the patient was transferred to our PICU.

All cultures and PCRs from blood and pericardial fluid were negative fir bacteria and viruses. Tumor markers were negative too. Bone marrow aspiration was negative for blood malignancies.

Cardiac MRI demonstrated thickened pericardium and a mediastinal, lobural and well-defined mass (max diameter $5 \mathrm{~cm}$ ), from the aortic arch to the left atrium and also another mass in contact with the lower part of the superior vena cava.

The patient underwent surgery which revealed that the masses were outgrowing from the parietal pericardium and infiltrated the left pulmonary artery and the left atrial auricle. The mass was partially removed. Surgical mass biopsies revealed EES of the pericardium. Staging CT scan showed that the disease was confined to the pericardium. The patient is currently undergoing chemotherapy, according to the paediatric Ewing's protocol.

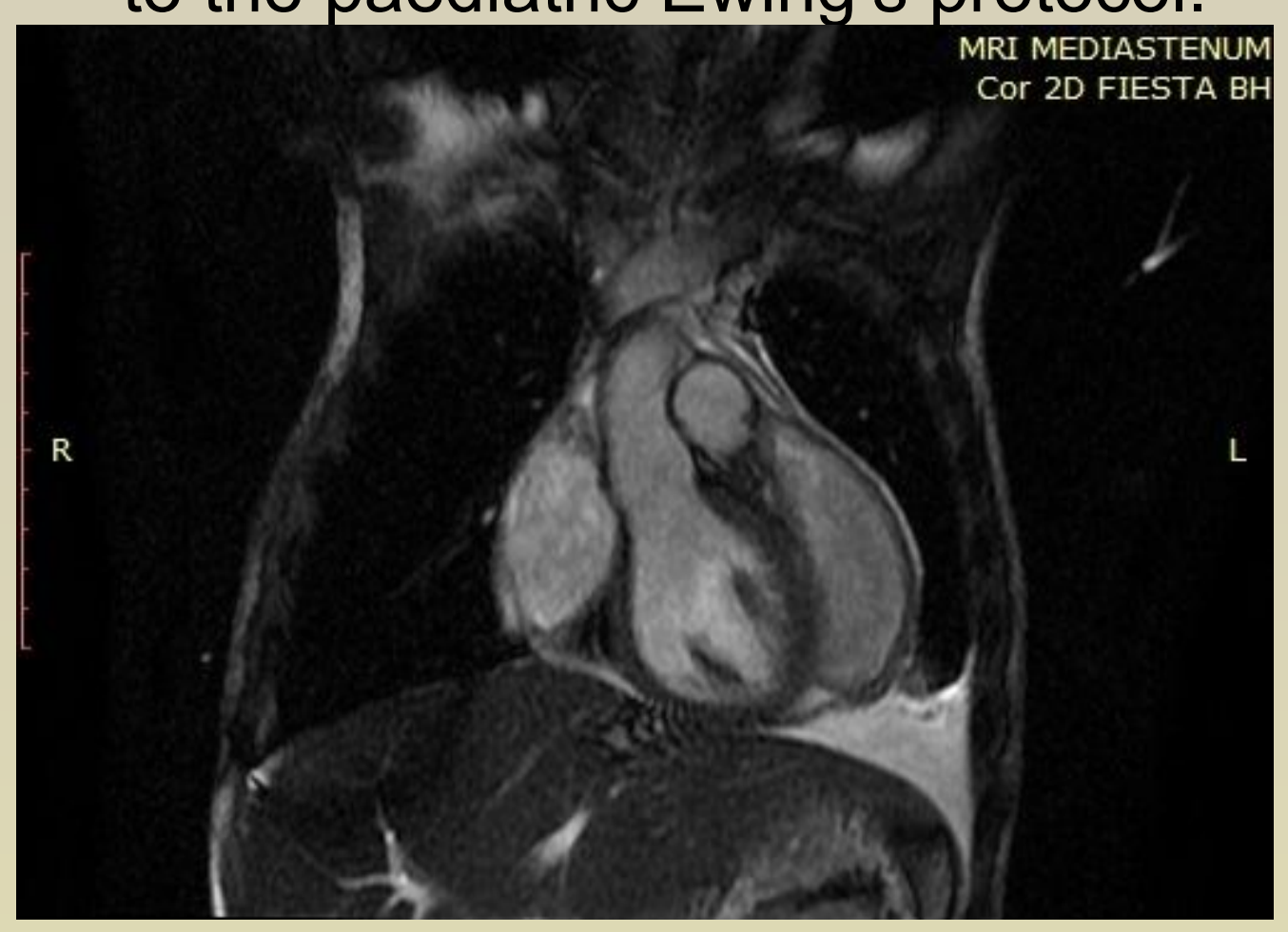

CONCLUSIONS

Primary cardiac neoplasms are rare in the paediatric population and the majority of patients remain asymptomatic until the tumor becomes large enough to cause signs and symptoms. Early diagnosis is of great importance for long-term survival.

\section{SOURCES}

1.Galyfos G, Karantzikos GA, Kavouras N, Sianou A, Palogos K, Filis K. Extraosseous Ewing Sarcoma: Diagnosis, Prognosis and Optimal Management. The Indiah Journal of Surgery. 2016;78(1):49-53.

2. Cash T, Mcllvaine E, Krailo MD, et al. Comparison of Clinical Features and Outcomes in Patients with

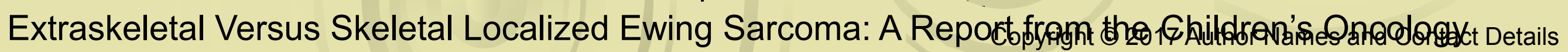

